\title{
The Effects of Number and Level of Restructuring in Insight Problem Solving
}

\author{
James N. MacGregor' and John B. Cunningham ${ }^{1}$
}

\begin{abstract}
Insight problem solving is characterized by restructuring. We hypothesized that the difficulty of rebus puzzles could be manipulated by systematically varying the restructurings required to solve them. An experiment using rebus puzzles varied the number of restructurings (one or two) required to solve a problem and the level at which the restructuring took place (sub-word level, word level, and supra-word level). Solution rates were higher for one restructuring than two, and higher for restructuring at the supra-word level (relation between words) than at lower levels. To explain the findings, we suggest that rebus puzzles initially engage normal reading processes, which involve extracting meaning at the level of relations between words.
\end{abstract}

\section{Keywords}

insight problem solving, rebus puzzles

'University of Victoria

The Journal of Problem Solving • volume 2, no. 2 (Fall 2009) 


\section{Introduction}

Solving a problem through insight is widely held to require a change in the problem's initial mental representation (Kaplan \& Simon, 1990; Knoblich, Ohlsson, Haider, \& Rhenius 1999; Metcalfe, 1986). For example, in the six matchsticks problem (Scheerer, 1963), the goal is to form four equilateral triangles from six matchsticks, and to do so requires switching from a representation in two-dimensions - which is the initial representation that people typically adopt-to one in three dimensions. The Gestalt psychologists introduced the term restructuring to describe this phenomenon. The resulting unexpected appearance of a solution in consciousness, together with the associated "Aha" feeling, has been termed the "insight experience," and an "insight problem" is one whose solution is likely to be accompanied by this experience (Smith, 1995).

A review of the recent literature reveals some evidence that there are different types of restructuring, but it remains unclear how to characterize the types. Only in the case of matchstick arithmetic problems (Knoblich et al., 1999), discussed below, does there appear to be theoretical and empirical grounds for believing that restructuring varies in identifiable ways. The present article extends this evidence into another domain, by investigating whether the difficulty of rebus puzzles varies with the number and the hierarchical level of the restructurings required for solution.

\section{Types of restructuring}

Ohlsson $(1984,1992)$ identified three different types of restructuring: elaboration, re-encoding, and constraint relaxation. Elaboration refers to a representational change brought about by supplying information to an originally incomplete representation. Re-encoding involves rejecting an initial, faulty interpretation, to reach a new interpretation of the problem. Thus, both elaboration and re-encoding involve the initial problem state, while the third mechanism, constraint relaxation, is defined in terms of change to an initially constrained or inappropriate representation of the problem's goal state.

While Ohlsson's work $(1984,1992)$ represented an important theoretical contribution, no empirical support for the three types of restructuring was provided. However, a further development and refinement of Ohlsson's theoretical approach, Representational Change Theory (RCT), proposed by Knoblich et al. (1999), has received empirical support. RCT hypothesizes both different types and different degrees of restructuring, and empirical evidence has been reported for both. The theory proposes that more than one constraint may be present at a time, and that a constraint with greater scope (affecting more of the problem representation) is less likely to be relaxed than one with lesser scope.Other things being equal, problems involving few constraints will be easier to solve than those that involve more, while those with constraints of narrower scope will be simpler than those where the critical constraints are of wider scope (Knoblich et al.).

The theory was tested using "matchstick arithmetic," where problems consisted of 
incorrect equations written in Roman numerals (I, II, III etc.), the operators + and - and equal signs $(=)$, constructed with matchsticks. The goal was to correct the equation by relocating one matchstick only. (For example, by changing IV = V - III into IV = VI - II.) Knoblich et al. (1999) identified three types of constraint in matchstick arithmetic, the value, operator and tautology constraints. The value constraint arises from an assumption that arithmetic values cannot be changed (such as moving a match to change $V$ to $\mathrm{VI}$ ). The operator constraint is the assumption that arithmetic signs are fixed (violated by moving a match to change - to + , for example). The tautology constraint arises because expressions like $X=X$ are usually meaningless (in arithmetic), and would be violated by changing $\mathrm{II}=\mathrm{II}-\mathrm{II}$ to $\mathrm{II}=\mathrm{I}=\mathrm{I}$, for example. They proposed that matchstick arithmetic problems are represented at three levels - numerals, functional terms, and equations - in order of increasing representational scope, and that the value constraint corresponds to the numeral level, the operator constraint to the functional term level, and the tautology constraint to the equation level. It follows that the probability of relaxing the three types of constraint should diminish in the same order. The results of several experiments supported this hypothesis.

While aspects of RCT have been tested using a different problem (Jones, 2003), the evidence for differences in difficulty of restructuring has been limited to the context of matchstick arithmetic problems. The issue of the generality of RCT as a theory of insight problem solving therefore arises. There is evidence from other contexts that insight problems vary in degree of difficulty, but this is not necessarily evidence for differences in restructuring, since problems may have sources of difficulty beyond those imposed by a requirement to restructure, such as the degree of mental lookahead required (MacGregor, Ormerod, \& Chronicle, 2001). Consequently, to further understand the restructuring process or processes, it will be helpful to identify whether there are different types or degrees of restructuring in other problem domains involving insight solutions. Below, we describe such a domain. We then derive and test predictions from Knoblich et al. (1999) in the context of that domain.

\section{Rebus puzzles as insight problems}

Previously, we reported evidence that solving rebus puzzles involves insight (Cunningham, MacGregor, Gibb, \& Haar, 2009; MacGregor \& Cunningham, 2008). A rebus combines verbal and visual clues to a common phrase, and solving a rebus typically requires breaking implicit assumptions of normal reading, similar to the restructuring required in insight. For example, "a front" (a bold front) requires attending to and explicitly interpreting the font characteristics of a letter or word. While font variations may carry figurative meaning, they are usually ignored in normal reading, rather than being interpreted literally, as is required to solve this example. A rebus based on a similar principle is "PUNISHMENT" (capital punishment). A single rebus may involve more than one such principle, and previ- 
ously we hypothesized that, the more there are, the more difficult a rebus will be to solve. This is consistent with RCT's hypothesis that, other things being equal, problems involving few constraints will be easier to solve than those that involve more.

We tested the hypothesis in a two-session experiment, where sessions were separated by approximately one week. In the first session, participants were presented with 21 rebuses requiring one, two, and three restructurings to solve. The results showed a significant linear decrease in solution rates across the three restructuring levels, from 70 percent correct at one restructuring to 43 percent at three. In the second session, participants tackled 24 rebuses, 8 at each of the three different degrees of restructuring. In this case, solution rates were significantly higher for one restructuring than for two or three, while the latter two did not differ significantly (MacGregor \& Cunningham, 2008).

In addition to partially supporting the "number of restructurings" hypothesis, the results supported the general proposition that solving rebus puzzles may involve insight, in several ways.Participants' rebus performance from both sessions correlated significantly with their mean performance on 20 Remote Associate (RAT) items, measured in the second session. In fact, the correlations of both sets of rebus scores with RAT scores were of the same order as the correlations of the rebus scores from the two sessions with each other, suggesting that the correlations with RAT were as high as the test/retest reliability of rebus puzzles would allow. In addition, rebus scores did not correlate significantly with general verbal ability, as measured by verbal analogy scores, suggesting that performance was determined by something beyond general verbal fluency. Finally, participants' rebus scores correlated significantly with self-rated insight.

A second study compared the performances of 172 participants on 10 spatial insight problems, 24 RAT items, 24 rebus puzzles, and a verbal test of fluid intelligence. Performance on rebus puzzles was found to correlate significantly more highly with both spatial insight and RAT performance than the latter did with each other. The findings suggested that rebus puzzles may incorporate elements of both spatial and verbal insight problem solving. In addition, rebus performance significantly predicted spatial insight problem solving after controlling for the effects of fluid intelligence and RAT performance (Cunningham et al., 2009).

\section{Predictions from RCT for rebus puzzles}

In developing predictions for matchstick arithmetic problems, Knoblich et al. (1999) hypothesized that the probability of a constraint being relaxed is inversely proportional to its scope. They suggested that, if problem representations are analogous to parse trees, and a constraint applies at a node in the tree, then the scope of a constraint will be determined by the height of the corresponding node in the parse tree. For example, constraints involving values are considered to be lower in the corresponding parse tree than those involving operators and should therefore be of lesser scope and have a higher probability of being relaxed.

- volume 2, no. 2 (Fall 2009) 
In many cases, the restructurings involved in solving a rebus appear to apply at different levels in a parse tree, and therefore translate directly into the scope concept as defined by Knoblich et al.(1999). For example, the rebus "you just me" (just between you and me) is likely to be read initially from left to right and result in what appears to be an anomalous phrase or sentence. To comprehend it as a meaningful phrase requires a restructuring at the level of a relationship between several words. In the language of constraint relaxation, it requires relaxing a constraint that word order in a phrase or sentence has syntactical meaning but not literal meaning. In other cases, a rebus is created by manipulating a single word in some way that has to be restructured, such as "raeg" (reverse gear). A constraint to be relaxed here is that single words are read from left to right. In still other cases, the required restructuring focuses on an individual letter or part of a word, such as "pPPod" (two peas in a pod), where one of the constraints to be relaxed is that letters are (usually) parts of words and not words themselves. These three examples appear to involve relaxing constraints that apply at different levels in a parse tree, with a constraint involving a relationship among words being higher in the tree than one involving a single word, which in turn is higher than one involving single letters or parts of words. The prediction from $\mathrm{RCT}$ is therefore that, other factors being equal, the relative difficulty of solving a rebus should decrease in this same order.

Previously, we found that rebuses requiring one restructuring were significantly more likely to be solved than those requiring two or three (MacGregor \& Cunningham, 2008). The result is consistent with Knoblich et al.'s (1999) prediction that a solution that violates more constraints should be more difficult than one that violates fewer. However, while number of restructurings may provide an approximate indication of a rebus's difficulty, it ignores the fact that not all restructurings are necessarily equal. Some may be more profound than others, requiring the suspension of more deeply-rooted or more widelyranging assumptions, and therefore be less likely to occur. The present experiment aimed to rectify this omission, by independently varying both the number of restructurings and their level in the parse tree.

\section{Experiment}

The experiment had two aims. The first was to test whether the difficulty of rebus puzzles varies with the number of restructurings required to solve them, when controlling for the scope of restructuring, where scope is defined as height in the corresponding parse tree (Knoblich et al., 1999). The second was to test whether the difficulty of a rebus increases with its height in a parse tree, as predicted by RCT.

Recently, we classified a sample of 152 rebuses in terms of the number of restructurings required to solve them (MacGregor \& Cunningham, 2008). In preparation for the present experiment, one of the authors classified the same rebuses in terms of the level of 
restructuring required-letter, word, or relation between words. In some cases, a decision was equivocal, and such cases were left unclassified. In other cases, where solving required more than one restructuring, the classification resulted sometimes in a mixed type, with restructurings at more than one level. For example "pPPod" (two peas in a pod) requires interpreting the letters PP as the words "two peas," interpreting "p--od" as the word"pod," and interpreting the words "two peas" as having the relation of being "in" the word "pod." Mixed types were initially omitted, leaving a set of 89 pure types.

We wished to manipulate both the number of restructurings and the level of restructuring. However, there were very few rebuses with more than two restructurings that were pure types in terms of the level of restructuring. For this reason, we limited the number of restructurings to either one or two.This left a total of 81 cases. We then randomly sampled from this set to achieve four in each cell of a $2 \times 3$ design with two levels of number of restructurings and three levels of restructuring level. ${ }^{1}$ Finally, an independent judge classified the 24 rebuses, guided by written definitions and examples of each of the three levels of restructuring. This independent classification agreed exactly with the original.

\section{Method}

Participants. The participants were 79 volunteers recruited at the University of Victoria. Fifty were female and 29 were male. Age information was not recorded, but all were adults.

Materials. We selected 24 rebus problems as described above. Problems were printed on $8 \times 11$ paper.

Design and Procedure. The experiment used a $2 \times 3$ within subjects design with two levels of number of restructurings ( 1 and 2 ), and three levels of restructuring level (letter, word, and relation between words).

We tested participants individually at the end of a larger procedure. The nature of the task was explained and they were given two practice items, each requiring restructurings at each of the three different levels. They then received a booklet with the test items and were allowed up to 12 minutes to complete them.

\section{Results and Discussion}

We assigned participants a score based on the proportion of correct answers. These scores ranged from .29 to .88 with a mean of .64 and $s=.13$. We analyzed the data using a repeated measures analysis of variance, with number of restructurings and restructuring level as independent variables. The results indicated significant main effects of number of restructurings, $\mathrm{F}(1,78)=231.24, \mathrm{MSe}=.04, \mathrm{p}<.001, \eta^{2}=.75$, and restructuring level, $\mathrm{F}(2,156)=64.72, \mathrm{MSe}=.04, \mathrm{p}<.001, \eta^{2}=.45$, and a significant interaction, $\mathrm{F}(2,156)=9.48$, $\mathrm{MSe}=.04, \mathrm{p}<.001, \eta^{2}=.27$.

The results are plotted in Figure 1, which illustrates the nature of the interaction effect.

- volume 2, no. 2 (Fall 2009) 
Figure 1. Mean Proportion of Rebuses Correct by Restructuring Level and Number of Restructurings (error bars show 95\% confidence intervals).

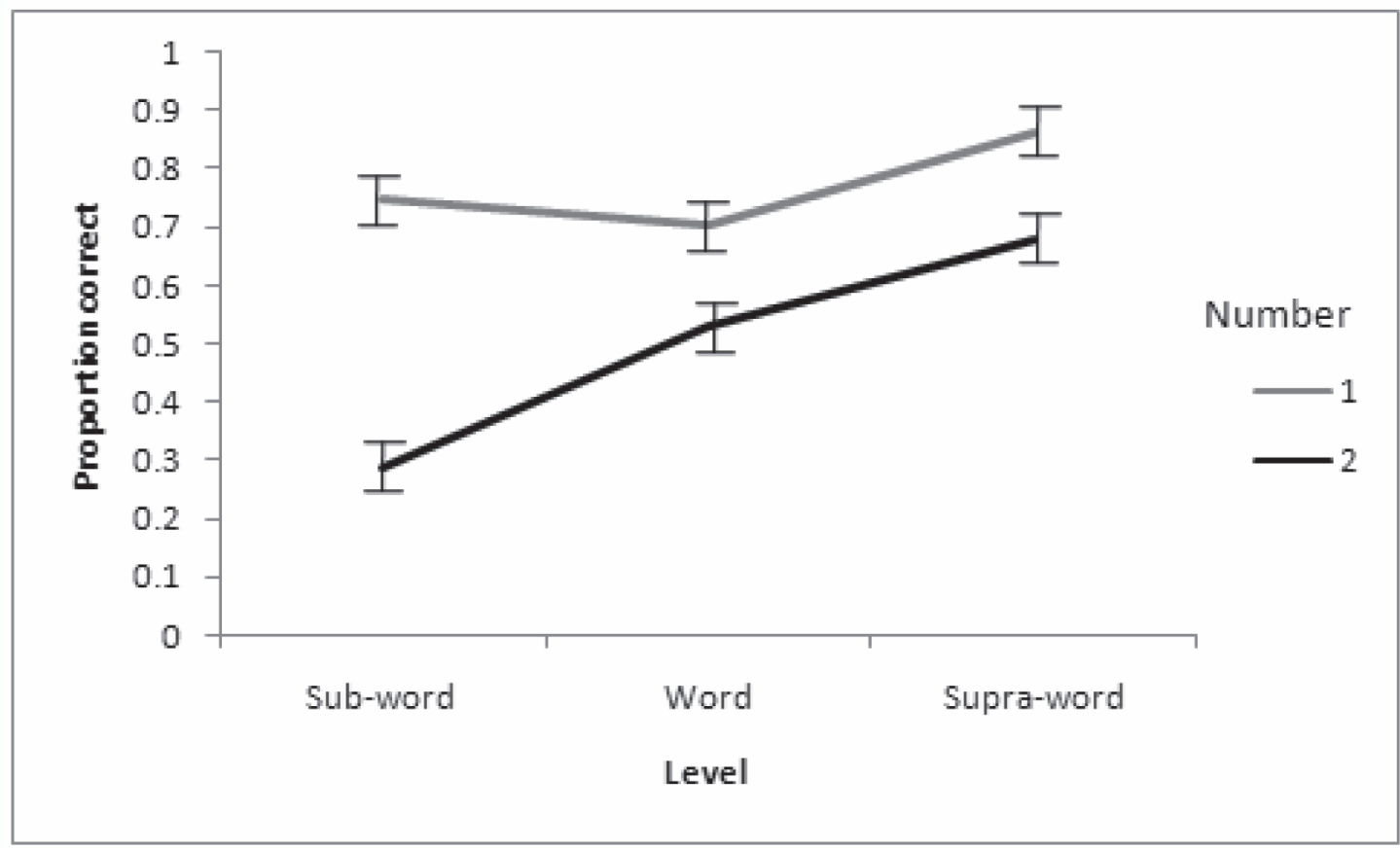

For rebuses requiring two restructurings there was an increasing linear trend in solution rates across levels of restructuring, from a solution rate of $28 \%$ at the letter level, through $53 \%$ at the word level, to $68 \%$ at the relation between words level. Planned comparisons indicated significant differences between each pair of means $(p<.05)$. For rebuses requiring one restructuring, however, there was no corresponding linear increase in solution rates, which were $75 \%, 71 \%$, and $86 \%$ for levels 1 through 3 , respectively. For rebuses requiring one restructuring, the solution rate for those operating at the letter level did not differ significantly from performance at the word level, $t(78)=1.39$, while the mean solution rate at the relation between words level was significantly higher than both.

The results for rebuses requiring two restructurings were completely opposite to the predictions from the scope hypothesis of Knoblich et al. (1999). In these cases, problem difficulty was inversely related to height in the parse tree, not directly related, as predicted from the definition of scope. The results for rebuses requiring one restructuring were also inconsistent with the scope hypothesis, in that those highest in the parse tree were significantly simpler than those at lower levels, not more difficult.

As can be seen from Figure 1, problems requiring one restructuring were simpler than those requiring two, at all three restructuring levels. This replicates our previous finding that rebuses requiring one restructuring are simpler than those that require more than one (MacGregor \& Cunningham, 2008). It also extends that finding, by showing that the result holds across different levels of restructuring. 


\section{General Discussion}

Insight problem solving is widely understood to involve a radical shift in a problem's cognitive representation, known as "restructuring." Recently, we reported evidence that solving rebus puzzles may involve restructuring, and that the difficulty of rebuses varies systematically with the number of restructurings required to solve them (MacGregor \& Cunningham, 2008). The present research sought to extend those findings, by answering two questions. First, does the difficulty of rebus puzzles vary with number of restructurings when controlling for scope of restructuring, as defined by Knoblich et al.(1999)? Second, does scope of restructuring influence the difficulty of a rebus?

The answer to the first question was affirmative. Solution rates for rebuses requiring two restructurings were significantly lower than those requiring one, at each of the three levels of the parse tree (sub-word, word, and supra-word levels).The results supported and extended our previous findings, and supported the hypothesis that the difficulty of an insight problem is a direct function of the number of constraints that need to be relaxed (Knoblich et al., 1999).

The second question asked whether the difficulty of a rebus is related to its scope, defined by Knoblich et al.(1999) as height in a parse tree. The results of Knoblich et al.supported the prediction that the difficulty of matchstick arithmetic problems would increase with increasing scope. In contrast, the present results did not support the prediction. In fact, the trend was in the opposite direction. For problems involving two restructurings there was a significant decrease in difficulty with increasing scope across all three levels of the parse tree. For problems requiring one restructuring, those at the highest level in the parse tree were significantly simpler than those at the two lower levels. The concept of scope, defined as directly related to height in a parse tree, does not therefore appear to generalize to the present set of stimuli.

There are several possible explanations for the present findings. One possibility is that initial attempts to solve a rebus engage the same processes as those used to comprehend normal text. While theoretical views on those processes differ, text comprehension is commonly understood to involve establishing relationships between concepts. This appears to be true of both structural and connectionist approaches to comprehension (Clifton and Duffy, 1998). In the present terms, this suggests a predisposition to search for meaning at the level of relations between words (supra-word level). As with insight problems in general, we assume this initial predisposition results in a search of a plausible but incorrect or incomplete solution space. At some point, through failure (Fleck \& Weisberg, 2004), or a failure to make progress (Kaplan and Simon, 1990; MacGregor et al., 2001), the search process shifts to a different search space. Whether or not this shift occurs by a conscious switch of attention (Kaplan and Simon, 1990), or through an unconscious redistribution of activation (Ash \& Wiley, 2006; Knoblich, Ohlsson, and Raney, 2001), we assume that it is

- volume 2, no. 2 (Fall 2009) 
more likely to move to a cognitively neighboring solution space than to a more distant one (MacGregor et al., 2001). The rebus solver is, therefore, likely to search in alternative "relation of words" level search spaces before delving deeper, into the word or sub-word levels.

If rebus puzzles initially engage normal reading processes, then a second possible explanation is that those puzzles that preserve whole words may offer more effective retrieval cues than those that do not. This explanation suggests that puzzles at the word and supra-word levels should, other factors being equal, be of equivalent difficulty, which was not what the findings showed. Nevertheless, it is clear that further research is required to fully identify the factors underlying the present pattern of results. A critical question that could be asked is whether solving the present rebus puzzles involved insight? Unfortunately, with very few exceptions, this is a criticism that applies to most studies of insight, where often the only justification for using an "insight" problem is that it has been used as such in the past (Weisberg, 1996). In contrast, there has been some effort to investigate whether solving rebus puzzles involves insight. One study found that rebus puzzle solving correlated significantly with performance on RAT items and with self-rated insight (MacGregor \& Cunningham, 2008). A subsequent study found that rebus performance correlated significantly more highly with both spatial insight performance and RAT performance than the latter two correlated with each other. In addition, rebus performance significantly predicted spatial insight problem solving after controlling for the effects of both fluid intelligence and RAT performance (Cunningham et al., 2009). In terms of existing standards, the evidence that rebus puzzles involve insight is therefore relatively impressive.

\section{Endnote}

1. As it turned out, there was only one candidate for the final cell, of two restructurings both at the level of relation between words. We completed this cell by randomly sampling three rebuses from those that required one restructuring at the word level and one at the relation between words level. 
Appendix 1. The rebus puzzles used (for solutions and classification, see Appendix 2).

\begin{tabular}{|c|c|c|c|}
\hline AGES & BAD wolf & PUNISHMENT & league \\
\hline R.P.I. & amUous & XQQME & $1 \mathrm{t} 345$ \\
\hline go stand & $\begin{array}{lll} & A & P \\
E^{S} & & P \\
& & \\
& U & A\end{array}$ & $\begin{array}{l}\text { person } \\
\text { ality }\end{array}$ & $\begin{array}{l}w \\
a \\
t \\
e \\
r\end{array}$ \\
\hline legal legal & $\begin{array}{c}\text { big big } \\
\text { ignore ignore }\end{array}$ & $\begin{array}{ll} & t \\
s & u \\
i & h \\
t & s\end{array}$ & $\begin{array}{l}\text { search } \\
\text { and }\end{array}$ \\
\hline$\frac{\text { somewhere }}{\text { rainbow }}$ & a home home & \begin{tabular}{|c} 
beating \\
beating bush beating \\
beating
\end{tabular} & rodiamondugh \\
\hline $\begin{array}{l}\text { little LARGE } \\
\text { little LARGE } \\
\text { little little } \\
\text { little LARGE }\end{array}$ & $\begin{array}{c}\mathrm{J} \\
\text { FRIEND } \\
\mathrm{S}_{\mathrm{S}}^{\mathrm{U}} \\
\mathrm{T}\end{array}$ & $\begin{array}{l}\mathrm{L} \\
Y \\
\mathrm{I} \\
\mathrm{N} \\
\mathrm{G} \\
\mathrm{JOB}\end{array}$ & $\begin{array}{c}o \\
\mathrm{r} \text { clock c } \\
\mathrm{k}\end{array}$ \\
\hline
\end{tabular}

- volume 2, no. 2 (Fall 2009) 
Appendix 2. Rebus solutions and classification.

\begin{tabular}{|c|c|c|c|}
\hline Solution & Number & Level & Explanation \\
\hline Dark ages & 1 & Sub-word & interpret letters in bold font as word \\
\hline Big bad wolf & 1 & Sub-word & interpret capitalized letters as word \\
\hline Capital Punishment & 1 & Sub-word & interpret capitalized letters as word \\
\hline Little league & 1 & Sub-word & interpret size of letters \\
\hline Grave error & 2 & Sub-word & $\begin{array}{l}\text { letter anagram/acronym } \\
\text { interpret capitalized letter as "big"/interpret }\end{array}$ \\
\hline Ambiguous & 2 & Sub-word & (big) letter as part of word \\
\hline Excuse me & 2 & Sub-word & $\begin{array}{l}\text { interpret letters as word parts (twice) } \\
\text { interpret letter as word/identify as standing }\end{array}$ \\
\hline Tea for two & 2 & Sub-word & for missing element in number series \\
\hline Go stand in the corner & 1 & Word & interpret position of words \\
\hline Round of applause & 1 & Word & interpret orientation of word \\
\hline Split personality & 1 & Word & interpret dislocation of word \\
\hline Water fall & 1 & Word & interpret orientation of word \\
\hline Paralegal & 2 & Word & interpret count of words/ use homonym \\
\hline Too big too ignore & 2 & Word & interpret count of words as homonym (twice) \\
\hline Sit down and shut up & 2 & Word & interpret orientation of word (twice) \\
\hline Search high and low & 2 & Word & interpret position of word (twice) \\
\hline $\begin{array}{l}\text { Somewhere over the } \\
\text { rainbow }\end{array}$ & 1 & Supra-word & relation between words \\
\hline Home (away) from & & & \\
\hline home & 1 & Supra-word & relation between words \\
\hline $\begin{array}{l}\text { Beating around the } \\
\text { bush }\end{array}$ & 1 & Supra-word & relation between words \\
\hline Diamond in the rough & 1 & Supra-word & $\begin{array}{l}\text { relation between words } \\
\text { relation between words/relative positions of }\end{array}$ \\
\hline A little on the large side & 2 & Supra-word & words \\
\hline Just between friends & 2 & $\begin{array}{l}\text { Word/supra- } \\
\text { word }\end{array}$ & $\begin{array}{l}\text { interpret count of words as plural/relation } \\
\text { between words }\end{array}$ \\
\hline Lying down on the job & 2 & $\begin{array}{l}\text { Word/supra- } \\
\text { word }\end{array}$ & $\begin{array}{l}\text { interpret position of word/relation between } \\
\text { words }\end{array}$ \\
\hline Rock around the clock & 2 & $\begin{array}{l}\text { Word/supra- } \\
\text { word }\end{array}$ & $\begin{array}{l}\text { identify distributed word/relation between } \\
\text { words }\end{array}$ \\
\hline
\end{tabular}




\section{References}

Ash, I.K., \& Wiley, J. (2006). The nature of restructuring in insight: An individual-differences approach. Psychonomic Bulletin \& Review, 13, 66-73.

Clifton, C., \& Duffy, S.A. (2001). Sentence and text comprehension: Roles of linguistic structure. Annual Review of Psychology, 52, 167-196.

Cunningham, J.B., MacGregor, J.N., Gibb, J., \& Haar, J. (2009). Categories of insight and their correlates:An exploration of relationships among classic-type insight problems, rebus problems, remote associates and esoteric analogies. Journal of Creative Behavior,43, $1-22$.

Fleck, J.I., \&Weisberg, R.W.(2004).The use of verbal protocols as data:An analysis of insight in the candle problem. Memory and Cognition, 32, 990-1006.

Jones, G. (2003). Testing two cognitive theories of insight. Journal of Experimental Psychology: Learning, Memory, \& Cognition, 29,1017-1027.

Kaplan, C. A. \& Simon, H. A. (1990). In search of insight. Cognitive Psychology, 22, 373-419.

Knoblich, G., Ohlsson, S., Haider, H., \& Rhenius, D. (1999). Constraint relaxation and chunk decomposition in insight problem solving.Journal of Experimental Psychology: Learning, Memory and Cognition, 25(6), 1534-1555.

Knoblich, G., Ohlsson, S., \& Raney, G. E. (2001). An eye movement study of insight problem solving. Memory \& Cognition, 29, 1000-1009.

MacGregor, J. N., \& Cunningham, J.B. (2008). Rebus puzzles as insight problems. Behavior Research Methods, 40, 263-268.

MacGregor, J.N., Ormerod,T.C., \& Chronicle, E.P.(2001).Information-processing and insight: A process model of performance on the nine-dot and related problems. Journal of Experimental Psychology: Learning, Memory and Cognition, 27, 176-201.

Metcalfe, J. (1986). Feeling of knowing in memory and problem solving. Journal of Experimental Psychology: Learning, Memory, \& Cognition, 12, 288-294.

Ohlsson, S. (1984). Restructuring revisited. II An information processing theory of restructuring and insight. Scandinavian Journal of Psychology, 25, 117-129.

Ohlsson, S. (1992).Information-processing explanations of insight and related phenomena. In M.T. Keane and K. J. Gilhooly (Eds.), Advances in the psychology of thinking, (Vol. 1, pp. 1-44). London: Harvester Wheatsheaf.

Scheerer, M. (1963). Problem solving. Scientific American, 208, 118-128.

Weisberg, R. W. (1996) Prolegomena to theories of insight problem solving: A taxonomy of problems. In R.J.Sternberg \& J. E. Davidson, (Eds.), The nature of insight. Paperback ed., pp.157-196. Cambridge, MA: MIT Press.

Paper submitted on July 26, 2009.

The final version accepted on October 29, 2009.

- volume 2, no. 2 (Fall 2009) 